
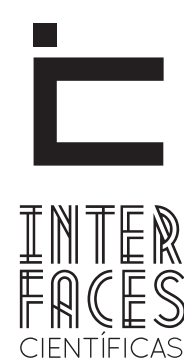

SAÚDE E AMBIENTE

\title{
CONHECIMENTO SOBRE POLIFARMACOTERAPIA DAS IDOSAS DO PROGRAMA DE ASSISTÊNCIA INTEGRAL À MELHOR IDADE EM ARACAJU
}

Juliana Leite Barbosa ${ }^{1}$

Carla Grasiela Santos de Oliveira ${ }^{3}$
Laianne Almeida Oliveira ${ }^{2}$

\section{RESUMO}

0 processo de envelhecimento acarreta, no idoso, alterações inerentes a senescência, bem como o surgimento de doenças crônico-degenerativas, o que leva ao constante uso de medicação. 0 uso concomitante de medicamentos pelos idosos pode ocasionar o surgimento de complicações, entre as principais estão as reações adversas e as interações medicamentosas. A pesquisa teve como objetivo geral verificar o conhecimento das idosas do Programa de Assistência Integral à Melhor Idade sobre polifarmacoterapia. Tratou-se de um estudo descritivo, transversal e de campo, com abordagem quantitativa, realizado na Universidade Tiradentes com o grupo de mulheres do Programa de Assistência Integral à Melhor Idade. A faixa etária que predominou entre as idosas foi de 61 a 70 anos. Dentre as doenças referidas a mais prevalente foi a hipertensão arterial. A maioria das idosas
(97,6\%) utiliza medicamentos e a polifarmácia foi verificada em 12,2\% da amostra. Em relação à classificação anatômica, a maioria das drogas medicamentosas consumidas correspondeu ao aparelho cardiovascular. Grande parte das entrevistadas (78\%) não sabe o que significa polimedicação e $97,6 \%$ sabem para que servem seus medicamentos. Percebeu-se assim, um bom conhecimento sobre os fármacos pelas idosas, apesar de não conhecerem o significado do termo polimedicação. Sugere-se realização de novas pesquisas sobre o tema, com enfoque na atuação do enfermeiro e nas orientações sobre o uso de medicamentos em idosos.

\section{PALAVRAS-CHAVE}

Enfermagem. Idoso. Polifarmacoterapia. 


\section{ABSTRACT}

The aging process causes changes in the elderly inherent senescence, as well as the emergence of chronic diseases, which leads to constant use of medication. Concomitant use of medications by the elderly can cause the onset of complications are among the leading to adverse reactions and drug interactions. The research main goal was to verify the polypharmacotherapy knowledge of elders in Program for Integral Assistance to the Best Age. This was a descriptive, cross-sectional field study with a quantitative approach, performed in Tiradentes University with the women's group of the Program of Integral Assistance to the Best Age. The age range that prevailed among the women was 61 to 70 years. Among the diseases reported, the most prevalent was hypertension. Most women (97.6\%) used medications and polypharmacy was found in $12.2 \%$ of the sample. In relation to the anatomical classification, the majority of medical drugs consumed corresponded to the cardiovascular system. Most of the women (78\%) did not know the meaning of polypharmacy and $97.6 \%$ know what they are for their medicines. It was noticed as well, a good knowledge of the drugs by the elderly, despite not knowing the meaning of the term polypharmacy. That further research on the topic, focusing on the work of nurses and guidelines on the use of medications in the elderly are performed is suggested.

\section{KEYWORDS}

Nursing. Elderly. Polypharmacotherapy.

\section{RESUMEN}

El proceso de envejecimiento hace que los cambios en las en esencia inherente de edad avanzada, así como la aparición de enfermedades crónicas, lo que conduce a un uso constante de la medicación. El uso concomitante de medicamentos de las personas mayores puede causar la aparición de complicaciones, y entre las cuales figuran las reacciones adversas e interacciones medicamentosas. El objetivo principal de la investigación fue verificar el conocimiento del Programa de mayores de Atención Integral a la Edad de Oro de poli fármacos. Tratase de es unestudio descriptivo , transversal y de campo con un enfoque cuantitativo, realizado en la Universidad de Tiradentes con el programa el grupo de mujeres Atención Integral a la edad de oro . El rango de edad que prevaleció entre las mujeres fue de 61 a 70 años. Entre las enfermedades reportadas la más frecuente fue la hipertensión. La mayoría de las mujeres( $97,6 \%$ ) utilizan medicamentos y polifarmacia se encontró en el 12,2 \% de la muestra. En relación a la clasificación anatómica , la mayoría de los fármacos médicos consumidos correspondió con el sistema cardiovascular. La mayoría de las mujeres, el $78 \%$ no conocía el significado de la polifarmacia y el 97,6\% sabe lo que son por sus medicamentos. Se observó también, un amplio conocimiento de las drogas por parte de la tercera edad, a pesar de no conocer el significado del término polifarmacia. Sugerimos la realización de nuevas investigaciones sobre el tema, centrándose en el trabajo de las enfermeras y las directrices sobre el uso de medicamentos en los ancianos.

\section{PALABRAS CLAVE}

Enfermería. Ancianos. La polifarmacia. 


\section{INTRODUÇ̃̃̃O}

O envelhecimento da população é uma condição natural de todo ser humano, portanto é irreversível e ocorre em todo o mundo. A população idosa tem crescido rapidamente e proporcionalmente no Brasil, onde é definida como idosa a pessoa que tem 60 anos ou mais de idade. Dentro desse grupo, as pessoas com idade acima de 80 anos, também, tem aumentado de forma acelerada e proporcional. Assim, constitui o segmento da população que mais cresce e corresponde atualmente a mais de $12 \%$ da população idosa (BRASIL, 2010).

A transição de uma situação de alta mortalidade e alta fecundidade para uma de declínio na fecundidade, ocorrida, simultaneamente, com uma crescente expectativa de vida conduz a um rápido processo de senescência da população brasileira. Sendo assim, reafirmando-se os estudos demográficos, ocorrerá um aumento da população idosa para os próximos anos (RIBEIRO; PIRES, 2011).

Um envelhecimento normal se associa a várias modificações que ocorrem na estrutura e nas funções dos principais sistemas fisiológicos. Alguns exemplos de alterações normais da senescência são a redução da massa muscular, redução da massa óssea, redução do conteúdo de água corporal, redução da capacidade aeróbica, dentre outros (MORAES, 2012).

Com o processo de envelhecimento aumenta a incidência das doenças crônico-degenerativas na população idosa, assim, esse grupo etário apresenta maiores probabilidades de desenvolver patologias múltiplas que necessitam de vários medicamentos. Dessa forma, a intervenção em saúde, mais frequentemente utilizada nos países desenvolvidos é a prescrição de drogas medicamentosas (BRICOLA et al., 2011).

A frequência de eventos adversos relacionados aos fármacos é maior neste grupo etário, aumentan- do expressivamente de acordo com a complexidade da terapia. É frequente o idoso apresentar de duas a seis receitas médicas e utilizar a automedicação com dois ou mais medicamentos, especialmente para aliviar sintomas como dor e constipação intestinal. Essa situação pode ocasionar eventos adversos, uma vez que o uso simultâneo de seis medicações ou mais pode elevar o risco de interações medicamentosas graves em até 100\% (SECOLI, 2010).

Diante dessa realidade, é importante que o profissional de saúde se atualize em relação aos grupos de medicamentos mais usados pelos idosos para que possa orientá-los quanto à administração de sua medicação, a fim de evitar o risco de reações adversas e interações medicamentosas, como também para que se tenha a melhora do quadro clínico e, consequentemente, melhora em sua qualidade de vida (ARAÚJO, 2011).

Uma política pública de saúde executada de forma bem orientada e voltada para a efetivação da saúde, oferece aos idosos, condições de viver naturalmente essa nova fase da vida. Durante o processo de senescência, a expectativa de todas as pessoas é a continuação da busca por uma vida saudável. Para isso, é relevante contar com as orientações dos profissionais da saúde, com serviços e ações de saúde que invistam na estruturação dessas condições de forma a promover a longevidade com qualidade (RIBEIRO; PIRES, 2011).

O estatuto do idoso reforça a obrigatoriedade constitucional do Estado na efetivação do direito à saúde, trazendo em seu bojo previsões direcionadas, de maneira que tal direito, no caso dos idosos, seja efetivado de forma plena e irrestrita (DIAS, 2011).

Com o aumento do consumo de fármacos que acompanha o envelhecimento da população, a polifarmácia nos idosos está se tornando uma normalidade na prática médica. A polimedicação é um 
termo definido como o uso concomitante e de forma crônica de múltiplos medicamentos (GALATO; SILVA; TIBURCIO, 2010).

Dessa forma, quanto maior for o número de medicamentos, bem como o tempo do tratamento e a dose prescrita, maior será o risco para o desenvolvimento das reações adversas e das interações medicamentosas. Sendo assim, o aumento da iatrogenia farmacológica não tem apenas a idade como fator de risco, mas também, a associação desta com a variedade de patologias e as alterações do metabolismo dos fármacos decorrentes das alterações do envelhecimento que cursam de maneira individual (SECOLI, 2010).

Segundo Bueno e outros autores (2009) é fundamental que os prescritores conheçam as influências de um medicamento sobre o outro, quando utilizados simultaneamente, sendo de importância o trabalho multidisciplinar, envolvendo diversas áreas da saúde, visto que o idoso apresenta situações emocionais e de saúde que se interrelacionam.

Sendo assim, torna-se importante verificar o conhecimento das idosas que fazem uso de vários medicamentos, no sentido de contribuir para com esse grupo etário e sua família, bem como, com os profissionais da área da saúde, para que avaliem suas práticas cotidianas relacionadas ao uso concomitante de medicação na população idosa, visando assim à prevenção de futuras reações adversas decorrentes da combinação terapêutica.

A pesquisa tem como objetivo geral verificar o conhecimento das idosas do Programa de Assistência Integral à Melhor Idade sobre polifarmacoterapia e como objetivos específicos: verificar o conhecimento das idosas com relação aos medicamentos que fazem uso, no tocante à prescrição, dosagem e posologia; identificar as possíveis dúvidas que as idosas apresentam relacionadas aos seus medicamentos e identificar as orientações da polifarmacoterapia prestadas por algum profissional de saúde.

\section{METODOLOGIA}

A pesquisa foi realizada na Universidade Tiradentes com o grupo de mulheres do Programa de Assistência Integral à Melhor Idade (PAIMI). O PAIMI funciona na sala 6 do bloco C, no Campus Aracaju Farolândia da Universidade Tiradentes, reservada para o desenvolvimento de diversos tipos de atividades oferecidos pelo programa. Tratou-se de um estudo descritivo, transversal e de campo, com abordagem quantitativa, realizada por meio de entrevista.

A pesquisa seguiu as diretrizes propostas pela Resolução n 196/96 do conselho nacional de saúde, com elaboração de um termo de consentimento livre e esclarecido (TCLE) assinado pelos sujeitos que concordaram em participar da investigação.

Os sujeitos da pesquisa foram todas as mulheres atendidas pelo PAIMI durante o período da coleta de dados. Os critérios de inclusão utilizados foram: pertencer ao grupo, aceitar participar da pesquisa e assinar o termo de consentimento livre e esclarecido. Como critérios de exclusão: não estar presente nas reuniões do PAIMI durante o período de coleta de dados ou não aceitar assinar o termo de consentimento livre e esclarecido.

A coleta de dados foi realizada após a aprovação do Comitê de Ética em Pesquisa (CEP) da Universidade Tiradentes sob o parecer 420.026 e a liberação da responsável pelo PAIMI com visitas semanais ao grupo para a aplicação de uma entrevista composta de 14 questões, abertas e fechadas, realizada individualmente com as idosas pelas pesquisadoras.

Os dados foram tabulados pelo programa SPSS 21.0 e as variáveis foram agrupadas e demonstradas por meio de gráficos e textos descritivos dos resultados encontrados. Os dados foram interpretados, discutidos e confrontados com a literatura, no intuito de responder os objetivos da pesquisa. 
A polifarmacoterapia foi definida pelo número de medicamentos usados concomitantemente pelas idosas, utilizando como referência o uso de cinco ou mais medicamentos (SECOLI, 2010).

Os medicamentos foram classificados pelo sistema de classificação Anatomical Therapeutic Chemical (ATC) da Organização Mundial da Saúde. Por esse sistema, os princípios ativos são divididos de acordo com o órgão ou sistema do organismo que atuam, e também de acordo com suas propriedades terapêuticas, farmacológicas e químicas. Assim, classificam-se em cinco níveis diferentes. No primeiro, estão classificados em 14 grupos anatômicos; no segundo em subgrupos farmacológicos/terapêuticos. 0 terceiro e quarto nível são grupos químicos/farmacológicos/terapêuticos e o quinto nível é o princípio ativo. Na presente pesquisa, os medicamentos foram classificados no primeiro nível da ATC (PIZZOL et al., 2012).

A pesquisa envolveu riscos mínimos onde os sujeitos envolvidos tiveram sigilo assegurado pelos pesquisadores e não passaram por nenhum tipo de constrangimento, já que, a realização da entrevista foi individualizada. Como benefícios, a orientação prestada sobre os medicamentos utilizados pelas idosas durante a realização da pesquisa.

\section{RESULTADOS}

Na presente pesquisa foram entrevistadas quarenta e uma idosas com idades compreendidas entre $58 \mathrm{e}$ 91 anos, estando $56,1 \%$ (23) na faixa etária dos 61 a 70 anos, encontrou-se assim uma média de idade de 69,6 anos.

Do total das idosas, $13(31,7 \%)$ eram casadas, 13 $(31,7 \%)$ viúvas, $10(24,4 \%)$ solteiras e $5(12,2 \%)$ separadas. Com relação à escolaridade, observou-se que $53,7 \%$ (22) das entrevistadas possuíam o primeiro grau completo. Quanto à renda familiar mensal preva- leceu a renda de 1 a 2 salários mínimos em 63,4\% (26) das idosas. Com relação à variável com quem mora, $75,5 \%$ (35) das idosas residiam com a família.

Quanto ao questionamento da existência de doença, 97,6\% (40) das idosas responderam possuir alguma alteração fisiológica (Figura1).

Figura 1 - Doenças prevalentes nas idosas pertencentes ao Programa de Assistência Integral à Melhor Idade. Aracaju/SE, 2013

\section{Doenças Prevalentes}

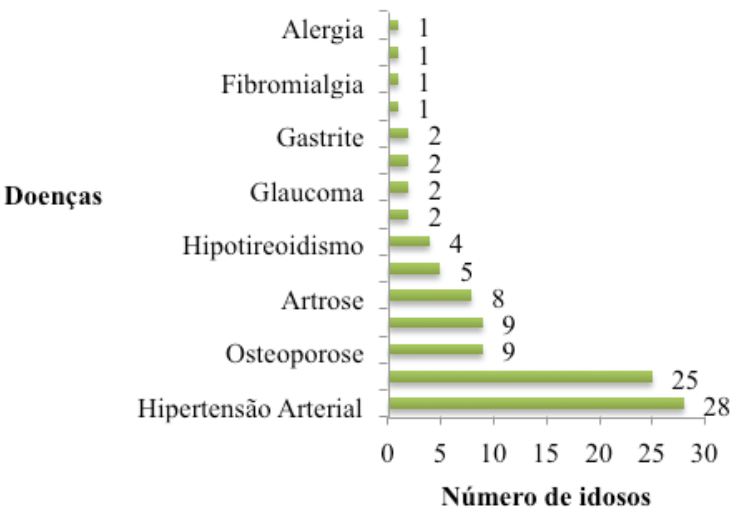

Fonte: Dados da pesquisa, 2013

Com relação ao uso de medicamentos na população pesquisada, 97,6\% (40) das entrevistadas responderam fazer uso de algum tipo de medicação (Figura 2). A polifarmácia foi verificada em $12,2 \%$ (5) da amostra. Os fármacos mais utilizados foram a sinvastatina, aproximadamente $26,8 \%$ (11) das idosas e em seguida a losartana, com aproximadamente 12,2\% (5) das entrevistadas. 
Figura 2 - Quantidade de medicamentos utilizados pelas idosas do Programa de Assistência Integral à Melhor Idade. Aracaju/SE, 2013

\section{Quantidade de Medicamentos}

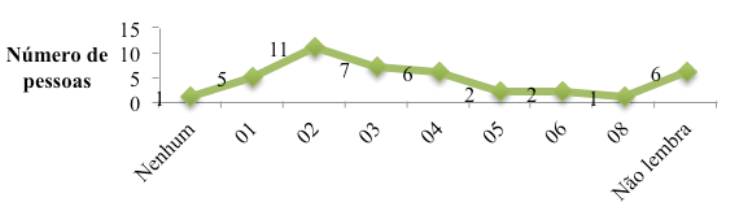

Quantidade de medicamentos

Fonte: Dados da pesquisa, 2013

0 número de medicamentos diferentes referidos pelas idosas foi de 56 fármacos (Figura3).

Figura 3 - Distribuição da quantidade dos medicamentos, segundo primeiro nível da Anatomical Therapeutic Chemical (ATC) utilizados pelas idosas do programa de Assistência Integral à Melhor Idade. Aracaju/SE, 2013

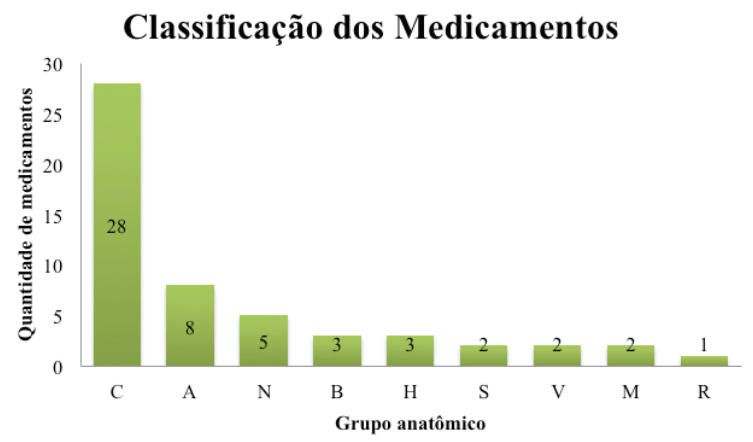

C - Aparelho Cardiovascular; A - Aparelho digestivo e metabolismo; N - Sistema Nervoso; B - Sangue e órgãos hematopoiéticos; H - Preparados hormonais; S - Órgãos dos sentidos; V - Vários; M - Sistema músculo-esqulético; R - Aparelho respiratório

Fonte: Dados da pesquisa, 2013

Na presente pesquisa, foi questionado às idosas se as mesmas sabiam o que significa polimedicação; como resultado $78 \%$ (32) das entrevistadas não sabem o significado da referida palavra. Já o restante respondeu que se trata de várias medicações.
Com relação à variável sabe para que serve seu medicamento $97,6 \%$ (40) das entrevistadas sabem a utilidade dos fármacos que fazem uso. Identificou-se assim um alto nível de conhecimento das idosas em relação à indicação do medicamento prescrito.

Com relação ao uso da medicação no horário correto, $78 \%$ (32) da população pesquisada respondeu tomar o medicamento no horário certo. Na variável como faz para não se esquecer de tomar a medicação, $78 \%$ (32) relataram outros tipos de alternativas, dentre essas prevaleceram como resposta: deixar em local de fácil visualização, adquirir hábito em tomar tal medicamento por isso não esquece, entre outras. Todas as idosas administram sozinhas seus fármacos.

No presente estudo com relação ao aparecimento de alterações no corpo após a administração do medicamento, $85,4 \%$ (35) das entrevistadas relataram não sentir nenhuma alteração, o restante referiu alguns sintomas como dor no estômago (2,4\%), tontura (4,8\%), enjoo (2,4\%).

Das quarenta e uma idosas entrevistadas, $87,8 \%$ (36) já receberam orientações sobre seus medicamentos por algum profissional de saúde (Figura 4) e $90,2 \%$ (37) receberam orientações que a polifarmacoterapia pode causar danos à saúde.

Figura 4 - Profissionais que prestaram orientações sobre os medicamentos às idosas do Programa de Assistência Integral à Melhor Idade. Aracaju/SE, 2013

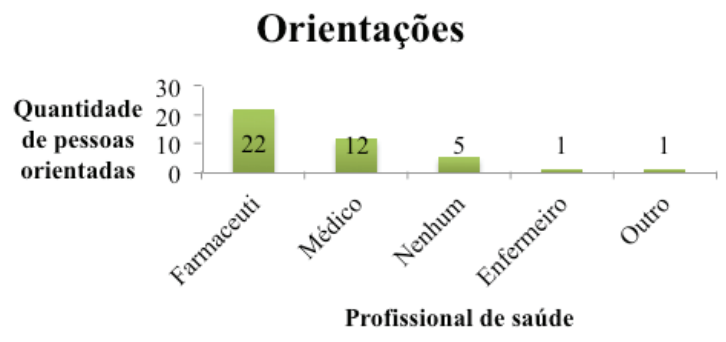

Fonte: Dados da pesquisa, 2013 


\section{DISCUSSÃO}

A população idosa, devido ao surgimento das doenças crônico-degenerativas, necessita do uso de vários medicamentos para garantir melhor qualidade de vida, sendo assim, a polifarmácia na maioria dos casos torna-se necessária (SANTOS et al., 2013).

Por meio da análise dos resultados obtidos no estudo, constatou-se a presença da polifarmácia em cinco idosas e o uso de apenas duas medicações pela maioria das entrevistadas.

As doenças que mais contribuem para a polifarmacoterapia são hipertensão, problemas cardiovasculares, problemas endócrinos e relacionados ao sistema nervoso central, sendo que as classes de medicamentos que mais contribuem são os anti-hipertensivos, antidiabéticos, psicotrópicos e antitrombóticos (GALATO; SILVA; TIBURCIO, 2010).

A hipertensão arterial sistêmica é uma das doenças mais comuns na população idosa, seguida pelo diabetes mellitus. E a sua prevalência pode chegar a 20\% nos idosos (ARAÚJO, 2011).

Em relação à existência de doenças prevaleceram na pesquisa a hipertensão arterial sistêmica, a dislipidemia, o diabetes e a osteoporose. A maioria das drogas medicamentosas consumidas correspondeu ao aparelho cardiovascular. Já os medicamentos mais utilizados que predominaram foram a sinvastatina e a losartana.

A elevada percentagem de utilização do grupo anatômico do aparelho cardiovascular na pesquisa está relacionada aos problemas de saúde que afetam esse sistema do organismo, apresentados pelas idosas como: hipertensão arterial, cardiopatia, dislipidemia, pós-infarto.

Foi verificado que a maioria das entrevistadas não sabe o significado da palavra polimedicação. Não foram localizados estudos mostrando o conhecimento de idosos sobre o significado da referida palavra.
Para Nassau (2009) além do uso de vários fármacos por si próprio, a dificuldade em gerenciar as medicações é um fator de risco para o idoso, podendo levar a sua hospitalização como já comprovado em pesquisas devido ao aparecimento de reações adversas, interações medicamentosas e iatrogenia.

Quando questionadas sobre o aparecimento de alterações no corpo após a administração do medicamento, a maioria das entrevistadas relatou não sentir nenhuma alteração.

Vários idosos tomam muitas drogas medicamentosas todos os dias para seus problemas de saúde. 0 envelhecimento na maioria dos casos muda a maneira de responder aos fármacos devido às alterações da farmacocinética. Dessa forma, quanto mais medicamentos o idoso toma, aumenta a probabilidade dos efeitos colaterais, como fraqueza, tontura, diarreia, náusea, constipação e outros efeitos complicadores (MACIEL, 2010).

Foi evidenciado na pesquisa que a maioria das idosas já recebeu orientações sobre seus medicamentos por algum profissional de saúde e também receberam orientações que a polifarmacoterapia pode causar danos à saúde.

Muitas vezes alguns profissionais não fazem a orientação de forma objetiva e eficaz para os pacientes, ou a falta de entendimento desses, pode ocasionar o surgimento de complicações como abandono do tratamento e interações medicamentosas ou alimentares, interferindo assim na garantia de uma melhor qualidade de vida dessas pessoas (BUSATO; LUNKES, 2012).

O profissional de saúde para prevenir e/ou tratar as complicações causadas pelo uso de vários fármacos em idosos deve conhecer as alterações fisiológicas do envelhecimento que alteram o metabolismo da droga; as doenças que mais acometem esse grupo etário; a farmacologia das medicações prescritas, 
bem como as possíveis interações medicamentosas e reações adversas; e as dificuldades encontradas pelo idoso com relação a sua terapêutica medicamentosa (SILVA; SCHMIDT; SILVA, 2012).

Além disso, para promover o uso racional de fármacos pelos idosos, outras medidas que devem ser adotadas são o esclarecimento aos familiares sobre as consequências do uso indiscriminado de drogas e o acompanhamento desse idoso pela equipe multiprofissional (SILVA, 2009).

\section{CONCLUSÃO}

Com a pesquisa realizada constatou-se que $97,6 \%$ (40) das idosas fazem uso de 02 a 04 medicamentos diariamente, sendo em sua maioria (50\%) medicações para o aparelho cardiovascular.

Observou-se que o profissional que prestou maior orientação sobre o uso correto das medicações foi o farmacêutico $(53,7 \%)$ e o enfermeiro foi citado apenas por $2,4 \%$ das idosas.

Dentre as doenças prevalentes nas idosas do PAIMI, a que mais se destacou foi a Hipertensão Arterial (68,2\%), seguida da Dislipidemia (60,9\%).

Assim, percebeu-se um bom conhecimento sobre os fármacos pelas idosas, apesar de $78 \%$ (32) não conhecerem o significado do termo polimedicação.

Sugere-se que sejam realizadas novas pesquisas sobre o tema com enfoque na atuação do enfermeiro e nas orientações sobre a polifarmacoterapia em idosos.

\section{REFERÊNCIAS}

Anatomical Therapeutic Chemical. Classification. Index with defined daily doses (DDDs). World Health Organization; 2010. Disponível em: <http://www.whocc. no/atc_ddd_index/>. Acesso em: 28 out. 2013.
ARAÚJO, C. L. Conhecimento de idosas sobre o uso de medicamentos e interação medicamentosa. Revista Brasileira de Ciências do Envelhecimento Humano, Passo Fundo, v.8, n.2, maio/ago. 2011, p. 188-195.

BRASIL. Atenção à saúde da pessoa idosa e envelhecimento. Ministério da Saúde. Secretaria de Atenção à Saúde. Departamento de Ações Programáticas e Estratégicas. Área Técnica Saúde do Idoso. Brasília, 2010.

BRICOLA, S. A. P. C. et al. Envelhecimento da população e a polifarmácia. Revista Eletrônica da Sociedade Brasileira de Clínica Médica, Regional São Paulo, v.1, n.1, Julho 2011.

BUENO, C. S. et al. Utilização de medicamentos e risco de interações medicamentosas em idosos atendidos pelo Programa de Atenção ao Idoso da Unijuí. Revista de Ciências Farmacêuticas Básica e Aplicada, Rio Grande do Sul, v.30, n.3, 2009, p. 331-338.

BUSATO, M. A. LUNKES, E. F. Assistência farmacêutica na Estratégia Saúde da Família em um município de pequeno porte de Santa Catarina. Revista Saúde Pública, Santa Catarina, v.5, n.1, jan-abr. 2012.

\section{DIAS, E. F. 0 Estatuto do Idoso e a judicialização} do direito à Saúde. [S.l.: S.n.], 2011. Disponível em: <http://jus.com.br/artigos/20304/o-estatuto-do-idoso-e-a-judicializacao-do-direito-a-saude>. Acesso em: 23 ago. 2013.

GALATO, D.; SILVA, E. S.; TIBURCIO, L. S. Estudo de utilização de medicamentos em idosos residentes em uma cidade do sul de Santa Catarina (Brasil): um olhar sobre a polimedicação. Revista Ciência \& Saúde Coletiva, v.15, v.6, 2010, p. 2899-2905.

MACIEL, F. P. Dificuldade do idoso na terapêutica plurimedicamentosa e seus efeitos colaterais - iatrogenia. Campos Gerais, MG. 2010. Disponível em: <https://www.nescon.medicina.ufmg.br/biblioteca/ imagem/2391.pdf>. Acesso em: 23 ago. 2013. 
MORAES, E. N. Atenção à Saúde do Idoso: Aspectos conceituais. Brasília: Organização Pan-Americana da Saúde. 201298 p.

NASSAU, F. M. Uso de medicamentos e Assistência de Enfermagem ao idoso hipertenso na Atenção Primária à Saúde: uma revisão da literatura. Belo Horizonte, MG. 2009. Disponível em: <https://www.nescon.medicina.ufmg.br/biblioteca/imagem/0399.pdf> Acesso em: 23 ago. 2013.

PIZZOL, T. S. D. et al. Uso de medicamentos entre idosos residentes em áreas urbanas e rurais de município no Sul do Brasil: um estudo de base populacional. Caderno de Saúde Pública, Rio de Janeiro, v.28, n.1, jan. 2012, p. 104-114.

RIBEIRO, A. P.; PIRES, V. A. T. N. Atuação do enfermeiro da Estratégia Saúde da Família na Atenção à saúde do idoso. Revista Enfermagem Integrada, I patinga: MG, v.4, n.2, Nov/Dez. 2011.
SANTOS, T. R. A. et al. Consumo de medicamentos por idosos, Goiânia, Brasil. Revista Saúde Pública, Goiânia, v.47, n.1, 2013, p. 94-103.

SECOLI, S. R. Polifarmácia: interações e reações adversas no uso de medicamentos por idosos. Revista Brasileira de Enfermagem, Brasília, v.63, n.1, jan/fev. 2010, p. 136-140.

SILVA, R.; SCHMIDT, O. F.; SILVA, S. Polifarmácia em geriatria. Revista da AMRIGS, Porto Alegre, v.56, n.2, abr-jun. 2012, p. 164-174.

SILVA, A. L. Estudo de utilização de medicamentos por idosos brasileiros. Belo Horizonte, 2009. Disponível em: <http://bvsms.saude.gov.br/bvs/premio_medica/2010/mencoes/trabalho_completo_anderson_ lourenco_silva.pdf> Acesso em: 23 ago. 2013.

1. Enfermeira pela Universidade Tiradentes. E-mail: fisiojulianaleite@ hotmail.com

2. Enfermeira pela Universidade Tiradentes. E-mail: lai_nane@hotmail.com

3. Enfermeira, mestre em Saúde e Ambiente, docente do curso de Enfermagem da Universidade Tiradentes. E-mail: carlagrasiela.enfermeira@ hotmail.com 